\title{
Magnetic resonance imaging in human teeth internal space visualization for requirements of dental prosthetics
}

\section{Marta Tanasiewicz ${ }^{1}$}

${ }^{1}$ Head of the Department. DMD, PhD, Department of Conservative Dentistry with Endodontics, Medical University of Silesia, Bytom, Poland.

Correspondence:

Marta Tanasiewicz

Plac Akademicki 17, 41-902 Bytom, Poland

e-mail: martatanasiewicz@sum.edu.pl

Tanasiewicz M. Magnetic resonance imaging in human teeth internal space visualization for requirements of dental prosthetics. J Clin Exp Dent. 2010;2(1):e6-11.

http://www.medicinaoral.com/odo/volumenes/v2i1/jcedv2ilp6.pdf

Article Number: $678929 \quad$ http://www medicinaoral.com/odo/indice.htm (C) Medicina Oral S. L. C.I.F. B 96689336 - eISSN: 1989-5488

eMail: jced@jced.es

\begin{abstract}
Objectives: The aim of this work has been a verification possibility to use 3D spin echo magnetic resonance imaging techniques in the dentistry within the scope of a dimensional imaging of the inner spaces of teeth during an prosthetic procedure.

Methods: 6 extracted molar teeth were used after preparation for the inner root canals fixations. Magnetic resonance (MR) measurements were carried out on a 4.7 T MRI system with Maran DRX console and home built actively shielded gradient coils and rf probe head.

Results: Research proceedings made it possible to compare the quality of internal tooth space after preparation for inner root canals fixations constructed using both classical methods (polymer mass impression) and non-imressional methods (MRI representation)

Conclusions: A numerical model of prepared root canals obtained with the method of the magnetic resonance visualization may constitute in the future a basis for a non-impressional technique of imaging, usefull for dental prosthetics. A numerical model of the prepared root canals obtained with the method of the magnetic resonance visualization imaging combined with the method of images analysis, may in the future constitute a basis for an automatic 3D modelling of the inner root canals fixations, supported with computer applications dedicated for the designing purposes (CAD-computer aided design).
\end{abstract}

Key words: Endodontic treatment, dental prosthetic, magnetic resonance imaging, spin echo technique. 


\section{Introduction}

In dentistry, during a complex medical procedure aimed at restoring damaged teeth, it is extremely important to create a correct and precise topographical image of the root canals. The necessary condition for a successful restorative procedure in dentistry is the precise mapping of the shape of dental cavities. Typically, impressional methods are used for that purpose. However, during the course of mapping, copying and storage, the impression material may change to some extent its shape and size, leading to imperfections in the process.

Visualisation of the root canals topography may help in estimation of the quality of mapping process. In the clinical practice visualisation has been restricted so far to $\mathrm{X}$-ray and optical imaging. Magnetic resonance imaging (MRI) has been used in research of healthy and decayed teeth during last decade. Several papers were presented showing usefulness of spin echo (SE) and gradient echo (GE) imaging, single point imaging (SPI), SPRITE and STRAFI techniques for visualization of the dental surface geometry as well as for distinction between soft tissue (pulp) and mineralized tissue (enamel, dentine and root cement) in the extracted teeth (1-6).

MRI techniques are currently being used in dentistry for diagnosis of temporomandibular joint diseases leading to a degeneration of the discs, inflammatory conditions of the facial skeleton, in examination of the salivary glands, maxillary sinuses, masseter muscles, in the detection of early bone changes such as tumors, fractures, inflammatory conditions and hematoma. MRI is also used to locate control points in the growth of the facial skeleton and in examining the bone before applying dental implants $(1,6)$.

In conventional MRI teeth are visible in the third lower quadrant of the head image and haven't been the main purpose of imaging until recently. The enamel and dentine should appear as dark streaks on all cross-sections, the pulp appears as a white or gray structure $(2,3,5,6)$.

Conventional techniques based on magnetic resonance do not allow for a realistic imaging of the internal tooth structure - the chamber and root canal (4). The pioneer publication treating about the application of magnetic resonance microscopy to image the structure of the tooth and the surrounding tissue is dated 1992 (1).

The obtained micro images $(100 \mu \mathrm{m} 3)$ made it possible to visualize the pulp and root canals, cavities and tooth structure. During the following years there appeared publications presenting attempts at using magnetic resonance microscopy to determine the geometry of the tooth surface, root canals, the location of cavities, and to compare tooth structure of younger and older patients (7). The techniques used for this type of imaging are generally referred to as spin echo and gradient echo methods and they facilitate the imaging of soft tissue or a hydrated medium (1-5).
The aim of this work has been an elaboration and a verification, whether there exists a possibility to use, on the level of in vitro analysis, those techniques of the MRI, which are based on the 3D sequence of the SE that may in the future find employment in the dentistry therapy and diagnostics within the scope of:

- A dimensional imaging of the inner topography of teeth and spatial structure of a chamber and root canals of teeth for the therapeutic and didactic aims.

- Introduction of a non-destructive and a non-impressional method of reconstruction of the topography of the inner spaces of the human teeth for the purposes of the reconstructive dentistry.

\section{Material and Methods}

Measurements were carried out in the Radiospectroscopy Laboratory of the Institute of Nuclear Physics of the Polish Academy of Sciences in Cracow.

Human molar teeth removed upon orthodontic recommendation were used during research. MR measurements were carried out on a $4.7 \mathrm{~T}$ research MRI system equipped with Maran DRX (Resonance Instruments Ltd.) console and home built actively shielded gradient coils and rf probe head. Echo time TE was $26 \mathrm{~ms}$. The dimensions of 3D data matrix varied from $64 \times 64 \times 64$ (low resolution image) to $512 \times 256 \times 64$ (high resolution image) in read, phase 1 and phase 2 directions, respectively.

In the first case it corresponds to a uniform resolution of $300 \mu \mathrm{m}$ in all three directions, in the second case to a resolution of $35 \times 63 \times 300 \mu \mathrm{m}$. The maximum resolution of $35 \mu \mathrm{m}$ was achieved in read direction because increasing the number of echo sampling points does not increase the total measurement time. High resolution image was acquired with two scans for every phase line in k-space to increase signal to noise ratio.

The repetition time of the measurements was $1,2 \mathrm{~s}$ and added up to a total of approximately 6 hours. The program 3D Segmentation was used to visualize the three dimensional structure of the teeth and reconstructions of the inner spaces of the human teeth.

Prior to the experiment all teeth were degassed in order to ensure uniform filling of the tooth chambers with the solution and to remove air micro bubbles. The degassing process is carried out by decreasing air pressure over the sample placed in a liquid. Gas bubbles increase their volume and float to the surface. After pumping them out atmospheric pressure is restored and the container (with internal diameter of $12,7 \mathrm{~mm}$ ) in which teeth have been degassed is sealed, then measurements are carried out. Degassing helps eliminate sources of artifacts created by the difference in magnetic susceptibility between air and the solution. 


\section{D Image Analysis}

Acquired data have been analysed using dedicated program developed for this purpose under IDL 5.5 (Research Systems) programming environment on $1.2 \mathrm{GHz}$ Pentium PC with Windows 2000 operating system. First, a median filter was applied to the $3 \mathrm{D}$ data to decrease noise without smoothing borders of the image regions with different intensities. Filtered data were then binarised (i.e. voxels intensities were set 0 or 1 depending on their values) and segmented into separated, compact $3 \mathrm{D}$ regions.

Algorithm for automatic segmentation of the 3D data is based on comparison of the absolute intensity of the neighboring voxels. The program allows also for visualisation of the data and the resulted segments in a form of pseudo-3D volumes, as well as for calculation of the segment's volumes (by calculating number of voxels in segments).

The segmentation algorithm was tested on numerical phantom of the tooth before applying it to the MR data. The phantom consists of 4 different segments mimicking the root canals, the tooth solid tissue, the outer liquid forming cylindrical volume, and the rest of the 3D cube. The two root canals were connected in its upper part only by few voxels. The algorithm properly divided the $3 \mathrm{D}$ data set into simulated segments and found their volumes.

\section{Statistical procedures}

Acquired data regarding tooth volume, internal tooth volume and amount of pulp in chamber and root canals were collected in samples with normal distribution. The samples' normality was verified with the Shapiro-Wilk test. The analyzed data was characterized using arithmetic mean, standard deviation and extreme values (minimum and maximum). The estimation of average values was given with 95\% confidence level. Samples including data regarding internal tooth volume obtained after pulp removal and treatment of inner tooth were treated as dependent. Mean internal tooth volumes after pulp removal and treatment were compared using the one-way ANOVA test (one-factor analysis of variance) carried out for the model with multiple measurements. The sphericity assumption was verified using Mauchley's test. The statistically significant result of the ANOVA test permitted post hoc comparisons, which were carried out using the Newman-Keuls test. The correlations' strength was evaluated using Pearson's correlation coefficient. All tests were carried out at significance level $\alpha=0,05$ using Statistica v 6,0 software (SUM, Katowice, Poland).

\section{Results}

Research proceedings made it possible to compare the quality of internal tooth space after preparation for inner root canals fixations constructed using both classical methods (polymer mass impression) and non-imressional methods (MRI representation). The phase of MR-based visualization of teeth after endodontic treatment, of negative polymer representations and of plaster models, demonstrated imperfections in the classical method of representation. Visual assessment of the analyzed material revealed imperfections in the impression-based method. Especially visible were deformations of the root canals, created in the process of making plaster models. The imprecise adherence of polymer resin to inner tooth surfaces after endodontic treatment should also be noted. During preparations for negative polymer representation, polymer resin residue inside the canals made it difficult to accurately image the root canals. This made it impossible to obtain an accurate image, and in consequence would have prevented the construction of an intracanal fixation fulfilling clinical requirements.

Research proceedings made it possible to create a spatial visualization and obtain numerical values characterizing the inner volume of chambers and root canals after treatment, the volume of negative polymer representations, and the inner volume of plaster models of chambers and root canals. Three dimensional reconstructions of the treated tooth, its negative polymer representation and plaster model are shown in figures $1 \mathrm{a}-1 \mathrm{~g}$. Numerical data is presented in Table I. Mean volumes of negative polymer representations and inner volumes of plaster models are statistically significantly different from the mean inner volume of teeth after endodontic treatment $(p=0,0259$ for polymer negative, $p=0,0255$ for plaster models). The difference between mean values of volumes of polymer representations and mean values of inner volumes of plaster models is not statistically significant $(p=0,6029)$.

\section{Discussion}

Research results presented in the analysis point to the possibility of applying MRI for visualizing tooth structure at the level of in vitro analysis (3-9). Research proceedings made it possible to compare the quality of representations of the inner tooth after treatment made using both classical methods (polymer mass impression) and non-impressional methods (MRI). Results show a discrepancy between values of parameters characterizing volume. The difference between volumes of the polymer negative and plaster model is visibly smaller (lower than 3\%) than the difference between volumes of the polymer negative and the inner tooth after treatment. This suggests that the transposition of inner tooth representation to the polymer negative leads to larger errors and might be responsible for potential imperfections of fixations constructed based on this technique, using the classical method. In most cases, the volumes of polymer negatives and plaster models are slightly smaller than the inner volume of treated teeth. Classical impression 


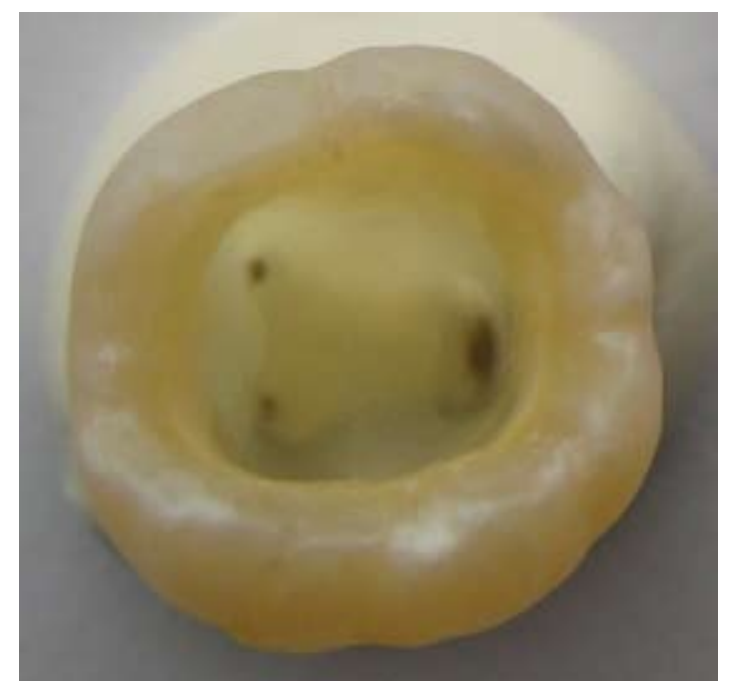

Fig. la Tooth after endodontic preparation.

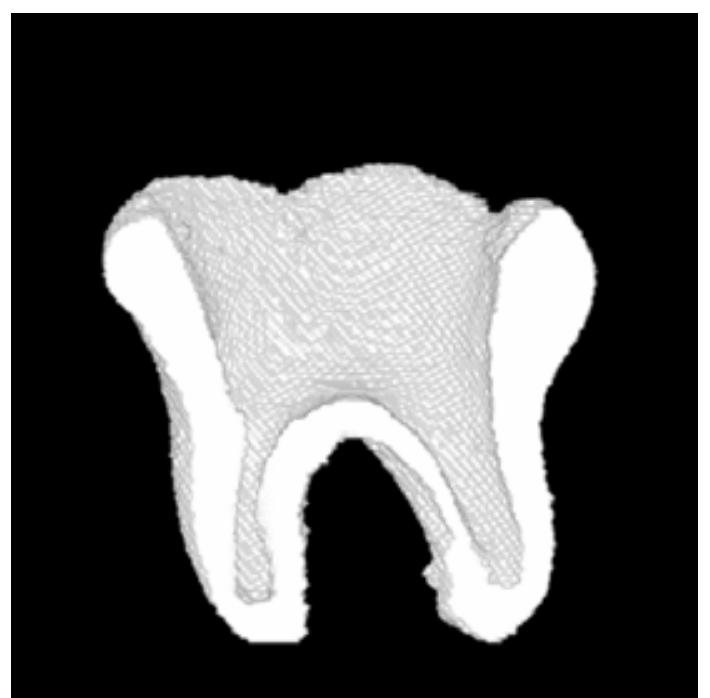

Fig.1b. Reconstructed representation of external and inner space of tooth

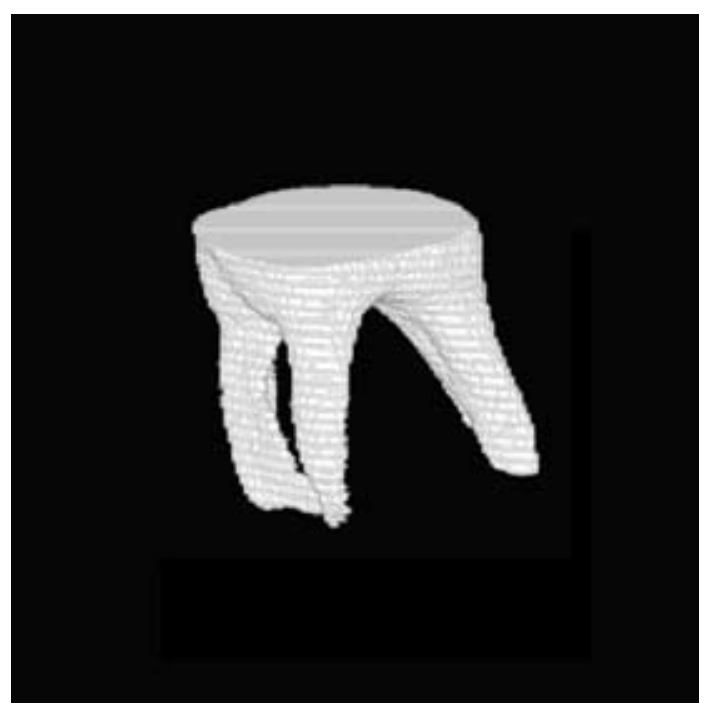

Fig. 1c. Reconstructed $3 D$ representation structure of inner space of tooth after endodontic preparation.

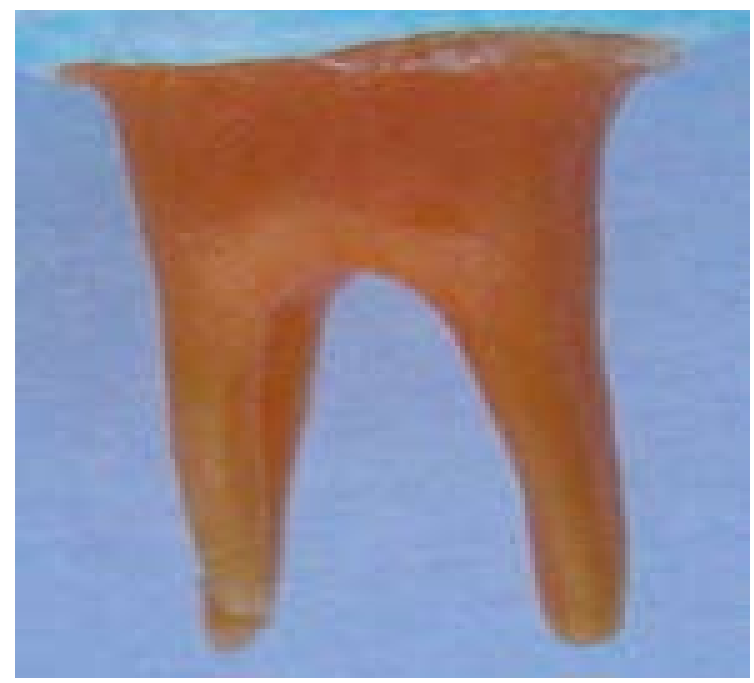

Fig. 1d. Negative polymer representations of inner space of tooth after endodontic preparation.

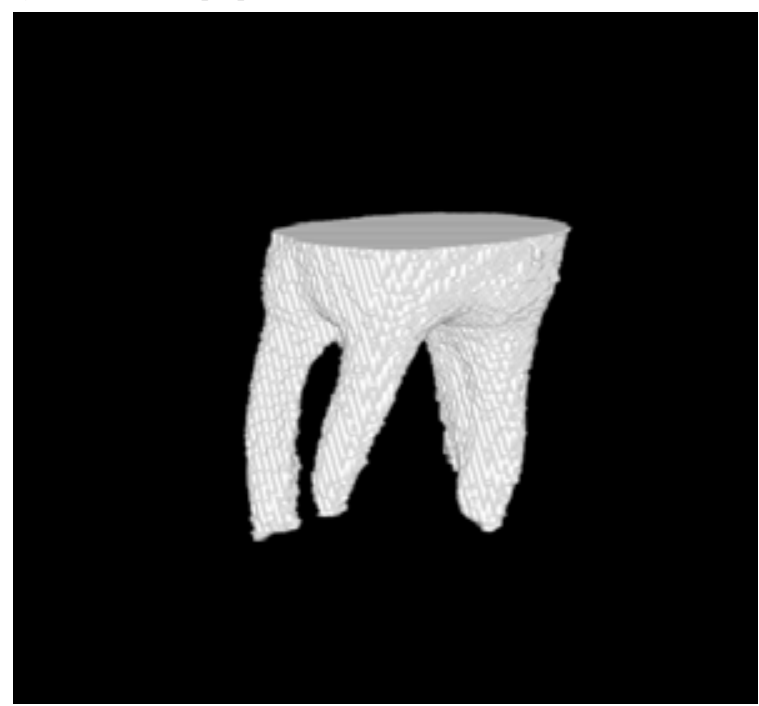

Fig. 1e. Reconstructed $3 D$ representation of negative polymer representations of inner space of tooth

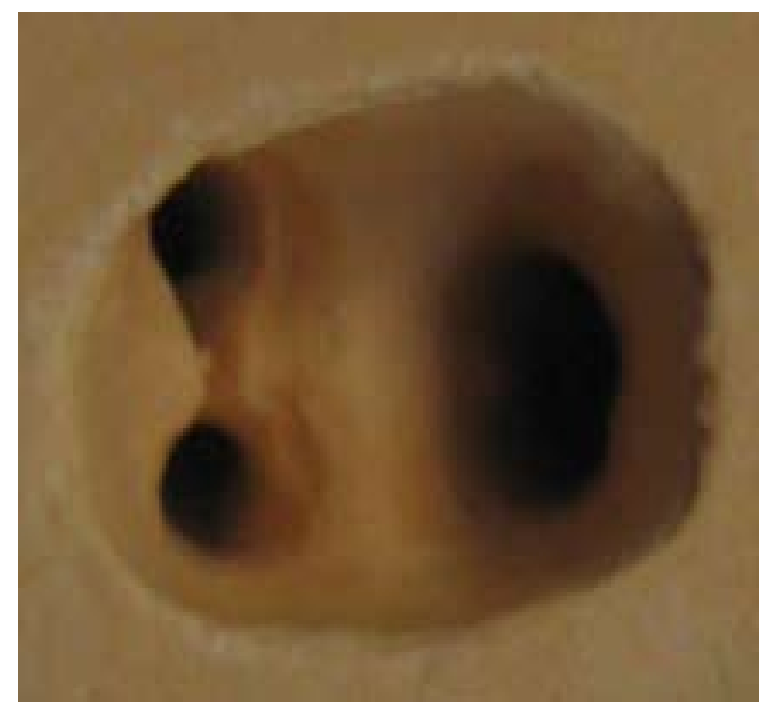

Fig. If. The plaster models of chambers and root canals of tooth. 


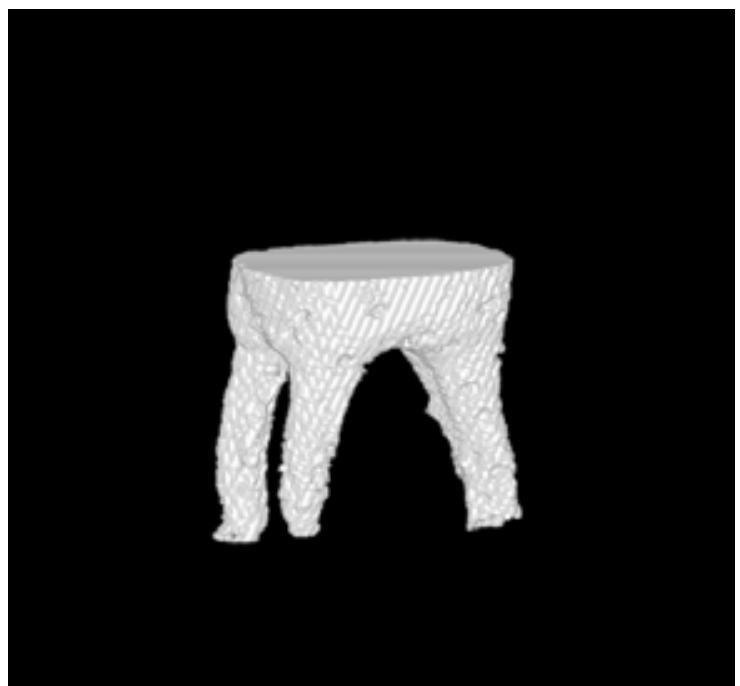

Figure $1 g$. Reconstructed $3 D$ representation of the plaster models of chambers and root canals of tooth. bed and applied systems of building three dimensional models representing cavities which make it possible to reconstruct lost fragments of crowns using an insertion with a designed and finely molded shape. Spatial representation of a cavity is obtained by scanning its shape with the use of industrial computed tomography.

Numerical data are then transferred to a system which designs the prosthetic restoration $(8,9)$. In future clinical operations the intracanal fixation could be milled from an appropriate block of any material based on an high-precision representation obtained using the MR method.

Realization of the established target and the achievement of satisfactory results, that may have a practical implications in the future, enabled to formulate the following conclusions:

The results that has been achieved, indicate the possibility to employ techniques of the MRI, that are based on

\begin{tabular}{|c|c|c|c|c|c|c|c|}
\hline \multirow[t]{2}{*}{ Variable } & \multirow{2}{*}{$\begin{array}{l}\text { Number } \\
\text { of test }\end{array}$} & \multirow{2}{*}{$\begin{array}{c}\text { Average } \\
{\left[\mathrm{mm}^{3}\right]}\end{array}$} & \multirow{2}{*}{$\begin{array}{l}\text { Standard } \\
\text { Deviation }\end{array}$} & \multirow{2}{*}{$\begin{array}{c}\text { Ratio of } \\
\text { variability } \\
\mathrm{V}[\%] \\
\end{array}$} & \multirow{2}{*}{$\begin{array}{c}\text { Range of value (min- } \\
\text { max) } \\
{\left[\mathrm{mm}^{3}\right]} \\
\end{array}$} & \multicolumn{2}{|c|}{$\begin{array}{l}95 \% \text { Partition of } \\
\text { trust }\end{array}$} \\
\hline & & & & & & $-95 \%$ & $+95 \%$ \\
\hline $\begin{array}{l}\text { Volume of inner space of teeth } \\
\text { after endodontic treatment }\end{array}$ & 6 & 125,46 & 8,60 & 6,85 & $114,10-135,46$ & 116,43 & 134,48 \\
\hline $\begin{array}{l}\text { Volume of negative polymer } \\
\text { representations of inner space of } \\
\text { teeth }\end{array}$ & 6 & 120,97 & 11,46 & 9,47 & $102,75-130,54$ & 108,94 & 132,99 \\
\hline $\begin{array}{l}\text { Volume of plaster models of } \\
\text { chambers and root canals of teeth }\end{array}$ & 6 & 120,07 & 9,91 & 8,26 & $103,28-130,0$ & 109,64 & 130,45 \\
\hline
\end{tabular}

Table I. Volume of inner space of teeth after endodontic treatment, negative polymer representations and plaster models of chambers and root canals of teeth.

materials (polymer mass) are subject to environmental changes and the durability of representations made with their use is dependent on external factors such as temperature, air humidity, transport and storage methods (1). Imperfections encountered in complex reconstructions are a result, among other things, of the instability of parameters of impression mass, which - due to storage and preparation conditions - undergoes changes in linear dimensions within a range normatively determined to reach a maximum level of $1,5 \%$ and deformations caused by pressure ranging from 0,8 to $20 \%$ (1). Another reason behind the difficulty of representation using classical methods is the topography and curvature of root canals in multiradicular teeth. Thanks to the developed MR visualization method a numerical model of the inner tooth was obtained which could, in the future, be also used in an automatic three-dimensional modeling technique using an appropriate computer aided design program designed for precise application of complex spatial models in reconstruction work. There currently exist descri- the 3D sequence of the $\mathrm{SE}$ as well as on the SPI, for the dimensional mapping of the outer topography of teeth as well as a structure of the root canals for the therapeutic and didactical purposes.

A numerical model of prepared root canals obtained with the method of the magnetic resonance visualization may constitute in the future a basis for a non-impressional technique of imaging, usefull for reconstructive dentistry. A numerical model of the prepared root canals obtained with the method of the magnetic resonance visualization imaging combined with the method of images analysis, may in the future constitute a basis for an automatic 3D modelling of the inner root canals fixations, supported with computer applications dedicated for the designing purposes (CAD-computer aided design).

\section{References}

1. Lloyd CH, Scrimgeour SN, Chudek JA, Hunter G, MacKay RL. Magnetic resonance microimaging of carious teeth. Quintessence Int. 1997;28:349-55. 
ce image and histology of human teeth. Proc Finn Dent Soc. 1992; 88:161-5.

3. Weglarz WP, Tanasiewicz M, Kupka T, Skórka T, Sułek Z, Jasiński A. 3D MR imaging of dental cavities-an in vitro study. Solid State Nucl Magn Reson. 2004;25:84-7.

4. Appel TR, Baumann MA. Solid-state nuclear magnetic resonance microscopy demonstrating human dental anatomy. Oral Surg Oral Med Oral Pathol Oral Radiol Endod. 2002; 94:256-61.

5. Beuf O, Seurin MJ, Briguet A, Lissac M. Magnetic resonance imaging of rodent teeth. MAGMA. 1999;8:83-6.

6. Olt S, Jakob PM. Contrast-enhanced dental MRI for visualization of the teeth and jaw. Magn Reson Med. 2004;52:174-6.

7. Ploder O, Partik B, Rand T, Fock N, Voracek M, Undt G, Baumann A. Reperfusion of autotransplanted teeth--comparison of clinical measurements by means of dental magnetic resonance imaging. Oral Surg Oral Med Oral Pathol Oral Radiol Endod. 2001;92:335-40.

8. Lee M, Yau HT. CAD/CAM use in the dental laboratory. Dent Today. 2006;25:88, 90, 92-3.

9. Wu L, Yan HX, Yang M. The reconstruction of geometric models of dentition defects based on 3-dimensional industrial computed tomography image. Shanghai Kou Qiang Yi Xue. 2006;15:535-8.

\section{Acknowledgements}

Supported by Grant no. 2 P05C 05426 from Ministry of Science and Informatisation of Poland. Author gratefully acknowledge the help of Dr Władysław P. Wegglarz from the Radiospectroscopy Laboratory of the Institute of Nuclear Physics of the Polish Academy of Sciences in Cracow in MRI research. 